\title{
De la notation musicale à la transcription de la prosodie : Description de la prosodie du français du XVle au début du XXe siècle
}

\author{
Schweitzer, Claudia ${ }^{1} \&$ Dodane, Christelle ${ }^{2}$ \\ ${ }^{1}$ Histoire des théories linguistiques (HTL), UMR 7597, Université Paris III - Sorbonne nouvelle et Université \\ Montpellier 3 (Département musicologie) \\ 2 PRAXILING, UMR5267, Université Paul Valéry - Montpellier 3 \\ claudia.schweitzer2@gmail.com, christelle.dodane@gmail.com
}

Résumé. L'existence de liens étroits entre musique et prosodie a poussé certains grammairiens à parler d'éléments musicaux pour désigner le rythme, l'accentuation et l'intonation de l'oral, ou encore, à avoir recours à la transcription musicale pour mieux représenter et décrire ces phénomènes. Dans la production de la parole, leur manifestation concrète est associée à l'évolution temporelle de la fréquence fondamentale, la durée et l'intensité. Ces variations sont perçues comme des changements de hauteur, de longueur et de sonie, que l'on retrouve également dans la musique. Il n'est donc guère étonnant que le vocabulaire utilisé pour décrire la langue parlée ou la musique se ressemble autant. L'Encyclopédie explique clairement que du rythme "naissent le nombre \& l'harmonie dans l'éloquence, la mesure \& la cadence dans la poésie », tandis qu'en musique, "le rythme s'applique à la valeur des notes, \& s'appelle aujourd'hui mesure ». Si nous étudions de plus près l'évolution de la transcription de la prosodie française entre le XVIe et le début du XXe, il est possible de dégager quatre grandes tendances. Au XVIe, les grammairiens se focalisent sur le fonctionnement des mots en contexte, au sein des différentes unités syntaxiques (Meigret, XVIe). Au XVIIe, des auteurs comme le grammairien Vairasse d'Allais ou le musicien Bacilly vont plutôt se focaliser sur un seul élément, la quantité, qu'ils utilisent pour parler du rythme, en travaillant notamment au niveau de la syllabe. Au XVIIIe, les Lumières cherchent à décrire et à expliquer avec précision le fonctionnement de la langue et de la musique, mais ils se heurtant au manque d'instrumentation. Ce manque sera comblé au début du XXe par les phonéticiens expérimentalistes (Rousselot, Roudet). Ces auteurs ont recours à la transcription musicale pour décrire avec une extrême précision l'évolution de la hauteur, de la durée et de l'intensité en utilisant une véritable partition, mais en négligeant toutefois les variations réellement pertinentes pour l'oreille. Dans l'ensemble, nous pouvons dégager un mouvement partant d'une description plutôt globale pour aboutir à une transcription extrêmement précise de la prosodie. En outre, chaque auteur, en fonction de son domaine, apporte des connaissances et un regard spécifiques. Dans cette communication, nous nous proposons donc d'analyser les apports de cette approche dans l'évolution de la description de la prosodie de la langue française.

\begin{abstract}
The existence of close links between music and prosody led some grammarians to use the expression of "musical elements" to designate rhythm, stress and intonation, or even to use musical transcription to better represent and describe this phenomenon. In speech production, their concrete manifestation is associated with the temporal evolution of the acoustic parameters of fundamental frequency, duration and intensity. These variations are perceived as changes in height, length and loudness, as this is the case in music. It is therefore not surprising that the vocabulary used to describe spoken language and music is quite similar. The Encyclopedia clearly explains that from rhythm emerge number and harmony in eloquence, measure and cadence in poesy, whereas that in music, rhythm applies to the values of notes, and is called today measure.
\end{abstract}




\begin{abstract}
If we examine more closely the evolution of the prosodic transcription of French between the sixteenth and the beginning of twentieth century's, we shall identify four major trends. In the sixteenth, grammarians focalized on the functioning of words in context, within various syntactic units (Meigret, XVIe). In the seventeenth, authors like the grammarian Vairasse d'Allais or the musician Bacilly rather focalized on quantity, which they used to describe rhythm, working on the level of syllable. In the eighteenth, "the Lumières" searched to describe and explain precisely the functioning of language and music, but had to face with the lack of instrumentation. This lack will be filled in the beginning of the twentieth century by experimentalists like Rousselot and Roudet. Indeed, these authors used musical transcription to describe, with an extreme precision, the evolution of height, duration and intensity using a proper musical score, but neglecting in the same time that some of those changes are not relevant for the ear. Overall, we identified a movement beginning from a more global description and achieving a highly accurate transcription of prosody. Moreover, each author, according to his field, brings specific knowledge and view. In this paper, we propose to analyze the contributions of this approach in the evolution of the description of French prosody.
\end{abstract}

\title{
1 Introduction
}

La prosodie désigne l'ensemble des aspects musicaux du langage, c'est-à-dire les paramètres de la fréquence fondamentale (sensation de hauteur), de durée (sensation de longueur) et d'amplitude (sensation d'intensité). Elle recouvre différentes fonctions comme le fait de véhiculer les affects, d'intervenir dans l'organisation sémantique ou encore d'être en partie impliquée dans la mise en œuvre de la syntaxe à l'oral (Vaissière, 1997). Cette décomposition en trois paramètres, la fréquence fondamentale, la durée et l'amplitude, analysables de manière instrumentale, est relativement récente puisqu'elle a été établie au cours du XXe siècle. En effet, les moyens techniques modernes donnent la possibilité de les visualiser très facilement sous forme de représentations temporelles telles que les spectrogrammes, les courbes mélodiques ou les courbes d'intensité. Mais on peut se demander comment faisaient les chercheurs pour décrire la prosodie avant l'apparition de ce type d'appareillage. Comme souvent, dans l'histoire de la grammaire, les auteurs avaient recours à des comparaisons, telles que des tableaux contenant les sons des différentes langues. Or, la comparaison entre la langue et la musique la plus naturelle en raison de leurs nombreuses similitudes. Mais pourquoi une telle comparaison nous semble aussi évidente ? Pourquoi a-t-elle pu s'imposer dès l'antiquité et a-t-elle été utilisée pendant des siècles par les grammairiens?

En musique, on parle de mélodie quand on évoque l'évolution de la hauteur au cours du temps, c.à.d. le passage d'une note à l'autre. Au niveau de la parole, on parle également de mélodie pour désigner la variation continue de la hauteur. Le paramètre d'intensité correspond autant dans la langue que dans la musique à des variations d'énergie. Enfin, la durée permet de décrire la longueur d'un son ou d'un ton en référence au retour périodique stable d'une unité donnée. On rejoint alors le domaine du rythme. En combinant certains ou l'ensemble de ces paramètres, on obtient les phénomènes d'accentuation qui se manifestent sous la forme de l'accent tonique ou métrique (en fonction de la structure de la langue ou de la musique) ou d'un accent expressif dans le but de transmettre un sentiment.

C'est en raison de ces similitudes que l'utilisation de la notation musicale s'est imposée auprès de grammairiens et des phonéticiens pour transcrire la prosodie. Cette représentation est très utile car elle permet de visualiser l'ensemble des phénomènes de hauteur, d'intensité et de durée sur un même support. En effet, avant l'apparition de la phonétique expérimentale à la fin du XIXe siècle, l'auto-perception était le seul moyen qui permettait de décrire la prosodie. Les grammairiens s'efforcent alors de décrire les sons qu'ils produisent eux-mêmes ou qui sont produits par d'autres personnes, et les sensations auditives associées à ces productions. Cependant, il va de soi que l'auto-perception est toujours soumise à la subjectivité de l'observateur, alors que l'instrumentation donne accès à une représentation objective. Ce mouvement se reflète dans le type de transcription choisi par les auteurs. Dans cet article, nous illustrerons cette évolution en réfléchissant à la façon dont les auteurs du XVIe au début du XXe siècle utilisent des éléments de la notation musicale pour transcrire la prosodie du français. Pour cela, nous les mettrons en correspondance avec les courants esthétiques dominants de chaque époque. Nous jugeons 
notamment intéressant de mettre en contexte les différentes accentuations et points de vue qui déterminaient les réflexions et recherches des grammairiens et la notation musicale de chaque époque pour comprendre comment les transcriptions des grammairiens étaient comprises en leur temps.

\section{Le XVle siècle : une attention portée sur le mot en contexte}

Qu'est-ce que la prosodie pour un théoricien du XVIe siècle ? Pour Ramus (1572), elle constitue, à côté de l'orthographe, une des parties essentielles de la grammaire :

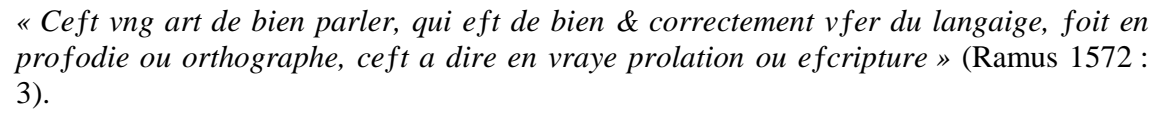

La prosodie est la réalisation verbale du texte, la mise en son des mots, des phrases, d'un discours selon des règles propre à chaque langue, et l'auteur indique un peu plus tard dans sa grammaire qu'il s'agit plus précisément du respect de la bonne quantité et de l'accent (1572 : 44), c.à.d. a priori de paramètres réalisés au niveau de la syllabe. L'essentiel de cette définition restera valable pour les grammairiens des prochaines générations. Pourtant, si nous portons un regard attentif sur les sujets traités par les auteurs de cette époque, nous pouvons rélever un aspect intéressant : l'attention des grammairiens porte notamment sur des ensembles d'éléments, ou, autrement dit, sur certains groupes de mots dans leur contexte syntaxique. Plusieurs aspects peuvent être concernés par leurs descriptions, tels que le rythme, l'accent et la mélodie.

\subsection{Le rythme ${ }^{1}$ et l'accent}

Quand les anciens grammairiens parlent du rythme, ils utilisent généralement des descriptions verbales. Meigret présente pourtant, dans son Tretté de 1550, une notation musicale: il se sert de portées, introduites dans le texte. L'objectif de l'utilisation de la notation musicale est une meilleure assimilation des explications verbales par une représentation visuelle qui donne à la fois des indications mélodiques et rythmiques (figures 1 et 2). Or, il paraît étrange que pour parler du rythme, Meigret n'utilise qu'une seule valeur de notes, la blanche. En outre, les mesures formées par les barres sont d'une longueur très inégale, incluant également des mesures ayant une valeur complètement inhabituelle à l'époque. Les exemples musicaux sont en effet moins conformes aux normes de notation en vigueur en musique qu'il ne le semble à première vue. Alors, à quoi servent-ils exactement ? En effet, ce sont les barres de mesure que l'on doit considérer comme étant au premier plan de cette notation. Si elles ne correspondent pas à des unités mathématiquement calculables, elles regroupent des ensembles de mots ou segments de la phrase. Ces barres ne servent point à accentuer par exemple la première syllabe (ou la première note) de la mesure comme c'est le cas dans une partition, au moins à partir du Baroque, mais elles forment des lignes de séparation qui correspondent aux points et aux virgules dans la langue écrite. En conséquence, le lecteur ne regarde pas un mot isolé, mais une unité de sens où, autrement dit, il met le mot en question dans son contexte. Quant aux notes, Meigret introduit une théorie selon laquelle la langue française présente de faibles mouvements mélodiques, déterminés par la structure des mots et leurs combinaisons. Nous allons montrer ce fonctionnement pour les monosyllabes, tout en soulignant que la théorie est plus complexe. En outre, après plusieurs chapitres consacrés exclusivement au comportement des monosyllabes dans différents contextes, l'auteur détaille son point de vue pour les autres types de mots avec la même précision qu'il ne le fait pour les monosyllabes.

Meigret commence par une réflexion sur la rencontre de deux mots monosyllabiques dans différentes combinaisons et positions de la phrase. Il constate alors :

\footnotetext{
"quand deux seuls monosyllabes se rencontreront au commencement d'une clause ou en suite d'un dissyllabe entier ou polysyllabe, que le premier sera élevé sur le second: comme ç'ęt mon maleur : si toutefois un dissyllabe ou polysyllabe les suit qui ait la première basse, le ton aigu sera au second monosyllabe, demeurant le premier grave: comme ç'ęt mon frere, ç'ęt mon compañon» (Meigret 1550 : 129, cf. figure 1).
} 


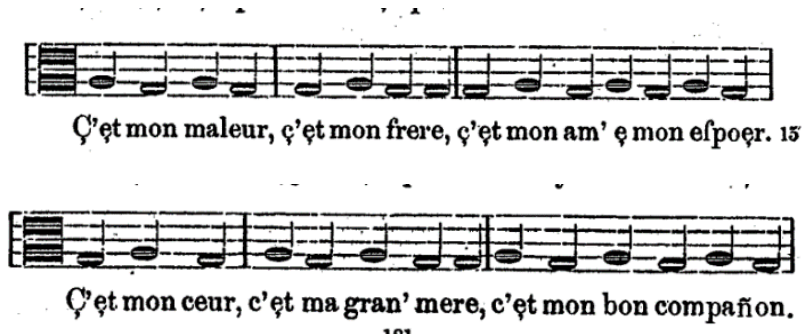

Figure $n^{\circ} 1$ : Transcription de l’inflexion mélodique selon Meigret (1550 : 129).

On le voit bien, Meigret amorce la description du fonctionnement de l'intonation d'une phrase musicale. Cependant, malgré l’utilisation de notes précises (on regarde la clé au début de chaque portée) ${ }^{2}$, les notes n'indiquent pas de mélodie au sens musical. Elles ne donnent qu'une relation approximative d'élévation ou d'abaissement de la voix. En effet, les explications données par le grammairien rendent plutôt compte de l'accentuation des mots qui, pour lui, va de pair avec une légère élévation de la voix. Autrement dit, l'accent se réalise par l'intonation. Les deux paramètres, bien séparés chez les phonéticiens modernes, ne le sont pas encore pour le grammairien du XVIe siècle. Dans un deuxième temps, l'auteur élargit le contexte. On passe à un niveau supérieur lorsqu'il n’y a que des mots comprenant un nombre de syllabes identiques. Ainsi, si le dernier mot d'un groupe est monosyllabique, l'avant-dernier mot est élevé, tandis que le reste de la phrase est prononcé sur la même hauteur (plus basse). S’il s'agit en revanche d'un mot à deux syllabes en dernière position, la dernière syllabe (voir la figure $n^{\circ} 2$, la syllabe « le » contenant un $e$ muet) reste sur le même ton.

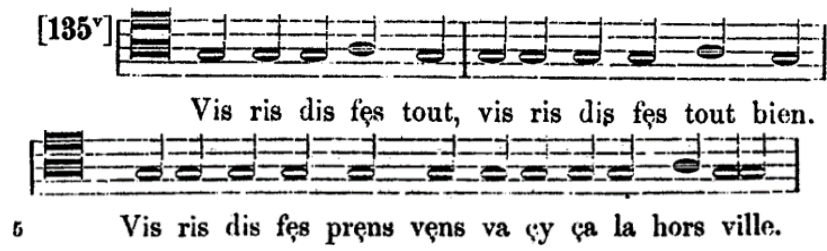

Figure $\mathrm{n}^{\circ} 2$ : Transcription de l’inflexion mélodique avec ou sans e muet (Meigret, $1550: 131$ ).

Les petites unités formées par les mots sont donc toujours soumises au fonctionnement d'un contexte plus vaste. Malgré l'apparence, Meigret traite a priori l'accent, et non la hauteur. La soit-disant mélodie qu'il indique est tout au contraire inhérente au mot (dans le sens où elle dépend du nombre de syllabes), et, dans un deuxième temps, soumise à un effet d'assimilation par la rencontre avec les mots qui le suivent.

\subsection{La mélodie}

Peu de remarques concrètes concernent la mélodie de la phrase chez les auteurs du XVIe siècle. Meigret mentionne l'élévation typique de la voix à la fin d'une phrase avec un point d'exclamation ou d'interrogation: "l'admiration élève la prononciation environ le commencement de la clause, là où l'interrogation le fait communément sur la fin: comme O l'home de bien! Qęl hom' ȩt' vous? » (1550 : 137). Le regard porté sur les exemples donnés a montré comment l'auteur se sert de la notation musicale pour illustrer les phénomènes accentuels. Le lecteur d'aujourd'hui peut être déçu de l'occasion, on dirait manquée, de noter par ce moyen la mélodie de la phrase. Le système de la portée laisserait de toute façon des possibilités pour dessiner avec plus de finesse les changements mélodiques sensibles dans les phrases prononcées. Toutefois, Meigret nous dit explicitement que son intention n'est pas là. Il lui importe au contraire de montrer que la langue française ne dispose que de très peu de mouvement mélodique :

\footnotetext{
"Or il me semble que pour plus facile doctrine, il sera bon de nous aider de quelque moyen oculaire pour mieux le faire entendre et donner quelque évidence à nos exemples: ce que me semble pouvoir être mené à fin, si en prenant quelque portion
} 
des notes de musique, nous nous en aidons par manière de prononciation élevée et abaissée, comme font les chantres, en élevant toutefois seulement ré sur ut par manière d'une modérée prononciation et non pas en chant, selon l'usage d'élever une syllabe sur l'autre exemple » (1550: 129).

S’agit-il d’un paradoxe ? Sans entrer dans les détails, on peut résumer que Meigret utilise la notation musicale pour indiquer les contours d'une mélodie qu'il sent dans sa langue, mais que cette mélodie n’a pas forcément une origine spontanée (il ne faut pas oublier que l'analyse du grammairien concerne la diction préparée, et non la parole immédiatement prononcée). Elle reprend cependant des observations que l’on peut faire facilement sur la langue parlée, bien que l'auteur ne les évoque pas :

- l'abaissement de la voix à la fin d'une assertion ;

- l'accent, localisé généralement à la fin de l'énoncé (assurée dans la notation de Meigret par l'élévation de la voix sur la syllabe pénultième) ;

- l'abaissement que le grammairien observe pour le $e$ « muet » d'une syllabe finale peut également être interprété comme une non-accentuation de la syllabe en question.

De cette théorie ressort clairement la dépendance des petites unités d'un contexte plus vaste. Elle semble sur ce point comparable aux règles de la musica ficta, système de notation musicale dans lequel les altérations doivent être ajoutées par le chanteur ou l'instrumentiste selon des règles repérables au seul moyen du contexte musical. ${ }^{3}$

Or, il nous semble qu'on peut aller encore plus loin en émettant l'hypothèse que l'utilisation de la notation musicale faite par Meigret s’intègre dans l'esthétique musicale propre à la Renaissance. Fondée notamment sur le contrepoint ${ }^{4}$, dans la majorité des compositions, on observe une conception linéaire, génératrice de lignes mélodiques. La chanson polyphonique française du XVIe siècle, inspirée par des thèmes narratifs, utilise notamment une forte accentuation des éléments rythmiques contenus dans les mélodies qui d'ailleurs souvent ne respectent que très peu le « rythme » des syllabes et des mots du texte. ${ }^{5}$ On pense donc en unités syntaxiques larges ou bien en sections. Meigret semble suivre cette conception, fondée sur le vaste développement de la phrase (musicale ou textuelle). La question peut alors se formuler comme suit : comment et dans quelle mesure peut-on considérer que la pensée musicale de l'époque se manifeste dans l'utilisation de la notation de la prosodie des auteurs ? L'hypothèse d'un tel rapport semble d'autant plus probable que la proximité entre la musique et la langue se voit souvent attestée chez les grammairiens comme chez les musiciens. En 1555 par exemple, Pontus de Tyard, dans son traité sur la musique, un texte typique de la pensée humaniste, parle de la longueur des syllabes du français. Il pose la question de savoir quels sont les critères pour reconnaître les syllabes longues et brèves et constate, tout comme les grammairiens, une différence entre les langues anciennes et le français. Le fait que la langue française ne fonctionne pas de façon quantitative constitue un important thème de réflexion, car le bon compositeur est évidemment tenu de trouver la quantité juste et convenable pour chaque syllabe.

C’est à la fin du siècle, que le nouveau rôle attribué à la dissonance et le coloris ajouté par les chromatismes ${ }^{6}$ change la perception. On est attiré par le détail, et l'attention des grammairiens comme des musiciens du siècle suivant, va porter davantage sur des éléments isolés que sur les unités syntaxiques. La définition détaillée de la prosodie, donnée par Jean Bosquet (1586 : 135-136), comparée à celle de Ramus quelques années plus tôt, semble indiquer ce changement. Selon Bosquet, la prosodie est :

«Vne partie de Grammaire, quy enseigne à nayuement prononcer, \& distinctement lire, \& accentuer chacune sillabe à sa mesure, quantité, \& bien-seance, comme apres, cornet, delie, fier, malle, mettre, matin, sale, Venus : lesquelz sans accens sont brefs, au regard de après, bâiller, cornette, delié, fier, mâlle, métre, mátin, salé, Vënus; esquelz les accens dénotent la prononcation, \& signification en deuoir estre autre. » ${ }^{7}$ 


\title{
3 Le XVIle siècle : la quantité au centre de l'intérêt
}

\subsection{Une concentration sur l'élément rythmique}

Ce sera alors un seul élément, la quantité, qui occupera largement les auteurs à partir du XVIIe siècle. Et cela, à un niveau de plus en plus limité et concentré sur des détails très fins. L'explication du grammairien Vairasse d'Allais en témoigne :

\begin{abstract}
«COMME on ne fauroit prononcer une letre ni une fyllabe, fans lui donner une quantité \& la faire breve, longue ou indifferente; par cette raifon la Profodie; eft naturellement la feconde partie de la Grammaire, puis qu'elle traite de la quantité des letres \& de fyllabes, d'où la Poëfie metrique a tiré fon origine. » (1681: 34).
\end{abstract}

La prosodie gagne en importance dans les réflexions des théoriciens, de sorte que chez Vairasse d'Allais, elle constitue toute la deuxième partie de sa grammaire. Il importe de constater que l'auteur la traite exclusivement au niveau des lettres et de la syllabe. Après les groupes syntaxiques et les parties de phrases, on s'intéresse maintenant aux unités les plus petites qui composent, selon la conception de l'époque, les mots ${ }^{8}$. La musique va former des mesures égales avec une hiérarchie interne des temps forts et faibles et un rythme a priori stable et égal, remplaçant la théorie complexe des proportions rythmiques qui était valable pendant la Renaissance ${ }^{9}$.

Dans le style baroque, le rythme prendra généralement une place primordiale : la langue est le modèle selon lequel se forme le chant qui, quant à lui, est imité par les instruments. Au bout du compte, on peut dire que la musique est calquée sur le modèle de la langue (d'où entre autres l'importance des styles nationaux et les querelles qui découlent de ces observations). La redéfinition théorique et son application pratique qui concernent la musique, constituent un long processus qui se terminera en France, beaucoup plus tard que dans le cas de l'Italie ou de l'Allemagne. Ce n'est qu'à partir des années 1660 que l'on peut parler d'un véritable style baroque pour la musique française et il n'est alors pas surprenant que dans le traité de chant publié par Bénigne de Bacilly en 1668, on rencontre encore les traces de la conception de Meigret selon laquelle le rythme des mots est traité à partir du nombre des syllabes qui la composent. On voit cependant que sous le couvert d'une présentation «traditionnelle », l'esthétique et sa théorisation changent. Bacilly (1668 : 330) commence par une argumentation visant à prouver que le français, « sans considerer s'il est joint à la Musique ou non », possède bien des syllabes longues et brèves. Il constate pourtant qu'à part l'existence de règles générales pour la quantité, il y en a d'autres qui ne sont valables que pour le chant. Le respect de la quantité naturelle des mots devenant de plus en plus important dans la composition musicale ${ }^{10}$, le chanteur doit être en mesure de corriger les erreurs du compositeur commises soit par négligence, soit par obligation de respecter les contraintes de la mesure, soit par le fait que les paroles aient dû être adaptées à une mélodie déjà existante (1668 : 332). L'apprentissage du chanteur se fait alors selon les catégories connues par le Tretté de Meigret. Les monosyllabes, dont le traitement constitue chez l'ancien grammairien la première étape de l'apprentissage, restent d'une importance primordiale, car c'est leur quantité avant tout qui peut varier dans un texte. Le musicien constate :

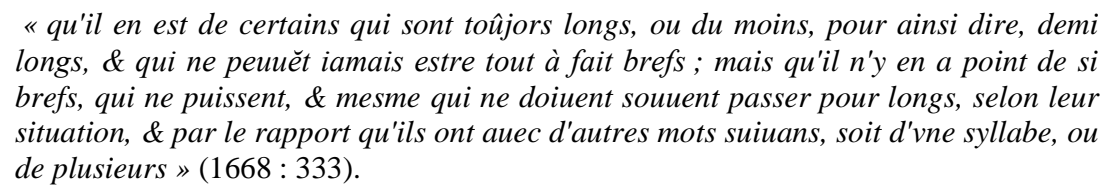

Peu de changement jusqu'ici. Le changement de l'accentuation selon le nombre de syllabes des mots dans un contexte donné que nous avons vu chez Meigret, réapparaît chez Bacilly qui le traite sous la notion de simetrie (1668 : 339). Cependant, l'accent est décrit chez l'auteur du XVIIe siècle par la quantité ${ }^{11}$ et non la mélodie. En tant qu'auteur écrivant un traité destiné à la pratique (du chant français), il établit deux règles pour expliquer quels faits gouvernent cette symétrie :

«Premierement, [...] tout mot feminin de deux syllabes a la penultiéme longue [...],

\& ce sans exception quelconque; \& tout au contraire la pénultiéme des mots 


\begin{abstract}
masculins de deux syllabes est toûjours brésve, à l'exception de certains mots qui se connoistront par la Table que i'en feray.

Secondement, il faut remarquer les Monosyllabes qui sont essentiellement longs ou demy-longs, \& qui ne sçauroient estre iamais brefs » (1668: 337).
\end{abstract}

La quantité intrinsèque à certains mots, ainsi que le caractère variable assuré par le $e$ muet à la fin des mots féminins sont d'une importance décisive. Ils participent à la création de la symétrie formelle, importante également dans la musique baroque ${ }^{12}$. On assiste à une période de transition pendant laquelle les anciennes valeurs restent acceptées, mais où elles sont complétées par d'autres explications et d'autres règles qui concernent un niveau subordonné, c.à.d. plus fin, de la phrase. Ces principes peuvent en outre être facilement appris par le public désirant comprendre la nouvelle esthétique musicale et notamment chanter en langue vernaculaire. En conséquence, Bacilly enchaîne un long chapitre intitulé « Moyens pour connoistre les Monosyllabes longs » avec des règles bien structurées, destinées à faciliter cet apprentissage. Suivent, dans la même optique, des chapitres sur la quantité des mots de deux syllabes féminins, ensuite masculins, et enfin des réflexions sur la quantité des finales masculines. ${ }^{13}$

\title{
3.2 Un changement épistémologique
}

Posons alors la question de ce qui se passe au fond de Meigret à Bacilly. La théorie, en surface, reste intacte, mais la présentation change de manière sensible. Certes, la hauteur ou la mélodie de la phrase à proprement parlé n'intéressent pas forcément un musicien : le chanteur ne choisit pas la mélodie qu'il chante, elle lui est donnée par la composition. Si elle servait à Meigret à parler de l'accent, Bacilly change de point de vue. Tout comme les grammairiens, il explique l'accent typographique qui exprime la hauteur chez les Anciens ${ }^{14}$, et le distingue expressément de l'accent réalisé par la longueur des syllabes longues et brèves, une distinction qui s'avérera importante pour les prochaines générations de théoriciens. On assiste alors à un transfert de l'ancienne théorie de Meigret dans un autre domaine, qui est conçu par les théoriciens du XVIIe siècle comme la distinction de plusieurs paramètres différents. Mais on reste également sur la distinction de paramètres stables (comme l'accent tonique inhérent au mot) et variables (la hiérarchisation des accents dans un contexte plus vaste), expliquée par Vairasse d'Allais comme suit :
«Les Grecs ont confondu le Ton \& l'accent fous le mot de tovnoÖ, bien que ces deux chofes foient fort differentes dans leur ufage. L'accent n'eft qu'une élevation de la voix toujours invariable dans un même mot: mais le Ton \& l'Emphafe font proprement des inflexions de la voix fujettes à varier felon les diverfes paffions \& circonftances que l'on veut exprimer. » $(1681: 51)$.

Quant à la musique, compositeur et chanteur sont censés connaître les syllabes longues, car, comme elles présentent un accent naturel, elles doivent se situer sur un temps fort dans la mesure. Avec le passage de la musique linéaire à une musique pensée plus verticalement ${ }^{15}$ et avec l'importance croissante des tensions créées par exemple par les dissonances, le regard se restreint. On peut établir une correspondance entre les travaux des grammairiens, où Vairasse d'Allais constate nettement que «LE fiege de l'accent eft la fyllabe fur laquelle on le pofe » $(1681: 40)$ et le développement observable chez les musiciens baroques. De la phrase, on passe à une unité plus petite, soit la syllabe, soit une suite d'accords déterminée par la tension dissonance et résolution. Il nous semble que le changement épistémologique observable chez les grammairiens s'inscrit dans la nouvelle esthétique musicale de l'époque. Les grammairiens n'hésitent d'ailleurs pas à continuer de se servir de la théorie musicale pour décrire les phénomènes de quantité. Ainsi on trouve chez Vairasse d'Allais l'explication suivante qui montre en outre la possibilité de combiner la longueur intrinsèque d'une syllabe donnée à la longueur due à l'émotion spontanée :

\footnotetext{
«Les fyllabes breves n'ont qu'un tems ou demi-note de mufique. Exemp. a-mi, o-beir, e-qui-t $\varepsilon^{\prime}$. Les fyllabes longues ont deux tems, ou une note entiere de mufique. Exemple, au-tant, im-pôt, guer-rier, \&c. [...] Mais les fyllabes longues par nature deviennent quelquefois trés-longues par pofition \& par accent, \& alors elles ont trois temps, ou une note \& demi de mufique. Ex. am-ple, re-gî-tre, an-tre, con-tre, neu-tre, crou-te, flû-te, \&c. » $(1681: 36,37)^{16}$
} 


\title{
3.3 Rythme, mélodie et emphase
}

Les indications concernant les autres paramètres qui composent la prosodie sont plus rares. On trouve par exemple un bref commentaire sur le rythme et sur la mélodie de la phrase dans les Remarques de Marguerite Buffet. D'après elle, ces deux paramètres dépendent de la ponctuation ou, autrement dit, de l'expression du sentiment réalisée par le locuteur :

\begin{abstract}
"Pour le ton de la voix il est facile de la bien regler quand on en est instruit : par exemple, la virgule arreste sans arrester, qui veut dire un instant. Le point final est bien different, il arreste en changeant le ton de la voix, soit de l'élever ou de l'abaisser, l'un est aussi bon que l'autre. » (1668: 152).
\end{abstract}

Vairasse d'Allais, comme nous l'avons déjà mentionné, traite également, à côté de la quantité et de l'accent, du ton et de l'emphase. Cette dernière se situe au niveau des mots et "n'eft autre chofe qu'un Ton remarquable qu'on donne quelquefois aux letres, aux fyllabes, aux mots \& aux fentences, dans une fignification extraordinaire. "(1681: 52). Il reste à noter que dans cette explication, l'accent est également localisé sur une unité plus petite : le mot.

\section{Le XVIIle siècle : la recherche d'explications détaillées et universelles}

\subsection{Un nouveau changement de perspective}

Les auteurs du siècle suivant continueront dans cette voie et les textes des grammairiens confirment largement le lien entre la prosodie et la musique. Grimarest désigne même la musique vocale comme " une espece de langue, dont les hommes sont convenus, pour se communiquer avec plus de plaisir leurs pensées, \& leurs sentimens. » (1707 : 195). D'Olivet constate quelques années plus tard que «la Musique [...] n'est, à proprement parler, qu'une extension de la Prosodie » (1737 : 9), une phrase que Beauzée commente, trente ans plus tard, comme suit :
«La musique n'est pas bornée à donner séchement la connoissance des différents tons \& de la valeur des notes; elle enseigne encore les diverses mesures auxquelles le chant peut être assujetti, le choix qu'il en faut faire selon la différence des pièces que l'on en compose, la proportion \& l'usage des soupirs, des demi-soupirs, des quarts des soupirs, \&c. C'est la même chose de la Prosodie. Elle ne doit pas seulement donner la connoissance des accents, \& fixer les degrés de la quantité des syllabes: elle embrasse encore naturellement tout ce qui peut résulter de la combinaison bien entendue de ces premiers éléments; les pieds \& leurs différents mêlanges, tant par rapport aux vers métriques dans les langues dont le génie s'est prêté à cette sorte de mélodie, que par rapport au rythme soit de la prose en général soit de la poésie des vers rimés. » (1767 : 161-162)

Les auteurs se reconcentrent de plus en plus sur une unité plus large que la syllabe. Le théoricien de la musique Raparlier montre par exemple cet élargissement de point de vue quand il parle de la phrase musicale : "On doit s'attacher à bien phraser son Chant, en reprenant haleine à propos, en consultant le sens des paroles qu'on doit chanter, \& sur-tout en faisant attention à la construction des phrases. » (1772 : 42). En conséquence, " on ne doit jamais reprendre haleine entre le Substantif \& l'Adjectif, ni entre le Verbe \& le Régime " (ibid.). La respiration qui marque une petite pause, souligne au fond la structure de la phrase entière. Les critères de hauteur ou d'intensité de la voix sont intégrés dans une théorie fine sur les accents. Le référent pour toute réflexion reste d'ailleurs l'écrit et on continue à insister sur la différence entre les langues mortes et le français en tant que langue vivante.

\subsection{La théorie de l'accent}

En principe, on distingue toujours l'accent imprimé ou typographique qui reflète un mouvement mélodique sensible dans le mot, de l'accent provoqué par des moyens intentionnés et mis en jeu par le locuteur. Dans la suite de D'Olivet, Beauzée divise les accents en accent prosodique (correspondant à une 
élévation et un abaissement du ton selon les accents imprimés) et en accent oratoire (« le principe \& la base de la déclamation », se référant à la mélodie et à l'intensité). Il donne l'astuce suivante pour distinguer les deux types d'accent : le premier modifie les syllabes une à une, relativement aux autres syllabes et il est invariable à l'intérieur d'une même langue ; le deuxième en revanche modifie toute la substance du discours relativement aux sens et aux différentes passions, et il varie selon les passions dont on parle (1767: chap. VI «De l'Accent des syllabes »). Ces explications reposent sur une nouvelle distinction entre la quantité physique (ou naturelle) et la quantité artificielle des syllabes : «La Quantité physique ou naturelle est la juste mesure de la durée de la voix dans chaque syllabe de chaque mot, que nous prononçons conformément aux lois du méchanisme de la parole \& de l'usage national » (1767: 120). Selon Beauzée, la durée des syllabes correspond aux nombres de un à cinq. On s'éloigne donc du système rythmique en vigueur en musique dans lequel les valeurs des notes simples correspondent traditionnellement à un système binaire. La quantité artificielle quant à elle concerne la versification et le rythme oratoire. La distinction entre les syllabes longues et brèves devient un «mécanisme »: "Une syllabe d'un mot est longue ou brève par le méchanisme, quand la voix sensible qui la constitue dépend de quelque mouvement organique que le méchanisme naturel doit exécuter avec lenteur ou avec célérité, selon les lois physiques qui le dirigent » (1767 : 123).

La quantité, la longueur ou la brièveté d'une syllabe, reste le facteur décisif pour décrire précisément le rythme de la phrase française. Chez D'Olivet, on trouve une explication qui essaie d'établir le lien entre la longueur et le temps que l'on passe sur chaque syllabe : «on met plus ou moins de temps à prononcer chaque syllabe, en sorte que les unes sont censées longues, \& les autres brèves : \& c'est ce qu'on appelle Quantité » (1736: 6). Cette quantité doit être soigneusement respectée par les musiciens. Si le récitatif est censé traduire et imiter la voix parlée, dans les airs mesurés, les accents du texte, naturels ou artificiels, doivent également être respectés dans la composition. Raparlier (1772 : 42) explique alors :

\footnotetext{
«On doit aussi s'attacher à la Prosodie, c'est-à-dire observer éxactement les longues \& les brèves ; sur-tout dans les Récitatifs. Dans les Airs mesurés, on peut observer la Prosodie, en augmentant ou diminuant la valeur des Notes, \& mettant l'équivalent sur celles qui précèdent, ou qui suivent, afin que la Mesure n'en souffre point ».
}

La mention du récitatif nous rappelle que c'est l'expression du sens d'un texte qui se trouve dorénavant au centre des réflexions. Comment traduire naturellement en musique ce que les hommes produisent spontanément lorsqu'ils parlent ? Quels moyens l'artiste a-t-il à sa disposition pour le faire ? Dans la volonté d'expliquer les phénomènes du monde jusque dans leurs moindres détails, les Lumières notamment essaient de décrire avec minutie ce qu'ils entendent, sentent et analysent.

\subsection{Mesurer la relativité ?}

Pourtant, comme il manque un outillage apte à mesurer, voire à enregistrer la voix (pour répéter plusieurs fois exactement la même réalisation d'un discours), l'ancien grammairien reste limité à sa perception et au jugement de son oreille. Les explications ne pouvant pas se baser sur des expérimentations mesurables, des chiffres ou des tableaux analytiques résultants, les auteurs établissent alors régulièrement des rapprochements relatifs. D'Olivet par exemple observe que «plus la prononciation est lente, plus la Prosodie devient sensible. On lit plus lentement qu'on ne parle; ainsi la Prosodie doit être plus marquée dans la lecture. » (1736 : 100). Mais dans quelle proportion ? Grimarest évoque précisément ce problème quand il explique que « cette mesure est à la vérité arbitraire, par raport à la lenteur, ou à la briéveté de la prononciation des personnes qui parlent, les uns le fesant avec beaucoup plus de facilité \& de promptitude que les autres » (1707: 25-26): on ne peut indiquer que la relation de la quantité des syllabes entre elles. Beauzée l'analyse comme suit :

«La Quantité des voix dans chaque syllabe ne consiste donc point dans un rapport déterminé de la durée de la voix à quelqu'une des parties du temps que nous assignons par nos montres, à une minute, par exemple, à une seconde, \&c. Elle consiste dans une proportion invariable entre les voix, laquelle peut être caractérisée par des nombres; en sorte qu'une syllabe n'est longue ou brève dans un mot, que par relation à une autre syllabe qui n'a pas la même Quantité » (1767 : 116). 
Ce faisant, on peut ré-établir le lien musical : dans une composition donnée, la durée des notes n'est jamais fixée, il est impossible d'en donner le temps en secondes ou minutes. Dans une composition baroque ou classique, la relation des valeurs des notes entre elles, est cependant nette : elle se calcule en proportions indiquées par les chiffres de mesure donnés au début et / ou au cours de la pièce. Cette proportion, si nette qu'elle puisse se présenter au XVIIIe siècle, ne satisfait pourtant pas forcément les musiciens ${ }^{17}$. En effet, sur la base des réflexions du physicien Joseph Sauveur, Etienne Loulié avait déjà publié en 1696 le modèle d'un " chronomètre » pour mesurer la pulsation. Le premier métronome au sens moderne ne sera cependant inventé qu'en 1812 par Johann Nepomuk Maelzel, établissant le lien entre la gradation et la subdivision de la minute. La quantité, au moins en musique, deviendra alors mesurable.

\section{De la fin du XIXe au début du XXe : méthode graphique et émergence de la phonétique expérimentale}

\subsection{Visualiser la mélodie grâce à la méthode graphique I accent tonique et accent oratoire en français ?}

Depuis le XVIIe siècle et les travaux d’Isaac Newton (1642-1727), l'idée s'impose progressivement que la science se constitue par des expérimentations répétées, qui permettent de dégager par induction des lois des phénomènes, que les théories rassemblent sous des principes directement tirés de l'expérience. Ces théories permettent de réaliser des prédictions, qui si elles sont vérifiées, confirment expérimentalement les lois et les théories. Ce schéma prend sa forme canonique dans la grande œuvre du XVIIIe, l'Encyclopédie, dirigée par d'Alembert et Diderot, dont l'objectif était de répertorier les connaissances et les savoirs de l'époque. Cette œuvre va fortement influencer le positivisme d'Auguste Comte (17981857 ) et notamment l'idée de l'unité et de l'organisation des sciences défendue par Saint-Simon (17601825). Au cours du XIXe siècle, les méthodes expérimentales scientifiques vont se développer. Elles seront également utilisées en sciences humaines et sociales et le développement de la méthode graphique par le médecin et physiologiste Etienne-Jules Marey (1830-1904) va marquer les débuts de la phonétique expérimentale à la fin du XIXe siècle. La méthode graphique est issue de la combinaison d'un cylindre enregistreur couvert d'une feuille de papier enduite de noir de fumée et d'un stylet qui vient y graver un phénomène vibratoire. Une embouchure est placée sur la bouche du locuteur et elle est reliée par un tube à l'inscripteur. Sur le tracé obtenu, on peut observer une suite de vibrations périodiques, de différentes formes et dimensions. Marey va appliquer cette méthode à la visualisation des sons de la parole, ce qui va marquer les débuts de la phonétique expérimentale.

Mais celui qui va produire les premières courbes mélodiques objectives à partir de la méthode graphique, c'est Hector Marichelle, professeur à l'Institut National des Sourds Muets de Paris et disciple de Marey. Il va calculer les variations de tonalité de la parole à partir de la forme des empreintes gravées dans les sillons du phonographe et les représenter sur une portée musicale adaptée ${ }^{18}$ sur laquelle nous reviendrons plus loin. Comme le faisait déjà Beauzée au XVIIIe (voir 4.2.), Marichelle fait la distinction entre l'accent tonique et l'accent oratoire. Selon lui, l'accent tonique est lié au rythme d'une langue donnée et il est indépendant de l'idée à exprimer et presque entièrement conventionnel. Il correspond à l'accent prosodique décrit par Beauzée. L'accent oratoire quant à lui correspond "au geste naturel que tout homme comprend sans initiation préalable » (Marichelle, 1897 : 110). Il ajoute que "l'accent oratoire, cette fleur de langage, exprime les idées générales communes à l'espèce tout entière; il laisse deviner les pensées délicates aux contours imprécis; il traduit enfin par des procédés invariables, dans quelque langue que ce soit, les fines nuances du sentiment, dont le mot simplement articulé ne représente que les formes vulgaires » (Marichelle, 1897 : 110-111). Selon lui, l'accent tonique est présenté successivement comme une élévation de la voix, puis comme un accroissement de l'intensité. Il se pose la question de savoir lequel de ces deux faits s’observe dans la réalité et pour lui, « seule la méthode graphique est en état de nous fournir à cet égard des renseignements positifs ». En ce qui concerne la représentation de l'évolution temporelle de la hauteur, Marichelle va modifier la portée musicale telle qu'elle existe à la fin du XIXe, de façon à marquer la différence entre les tons et les demi-tons (cf. figure 3). Il va ainsi diviser 
la portée en 10 lignes et 9 niveaux de hauteur, correpondant soit à un intervalle ${ }^{19}$ d'un demi-ton (niveaux plus étroits), soit à un intervalle d'un ton (niveaux plus larges). Chaque ligne correspond à une note de la gamme ( $\mathrm{mi}_{2}$ pour la ligne $1, \mathrm{fa}_{2}$ pour la ligne $2, \mathrm{sol}_{2}$ pour la ligne 3 , etc.). En utilisant un tableau de correspondances entre le nombre de périodes comprises dans $1 \mathrm{~cm}$ de sillon et chaque note de la gamme, lorsque la vitesse de rotation du cylindre est de $34 \mathrm{~cm}$, il est possible de construire la courbe représentative de l'évolution temporelle de la mélodie. Cette courbe est retranscrite sur le papier par une ligne continue, qui rappelle la notation d'un glissando ${ }^{20}$, une technique musicale destinée à réaliser un intervalle entre deux notes en glissant rapidement, en passant par toutes les notes intermédiaires et microintervalles possibles (toujours selon le type d'instrument) ${ }^{21}$. Marichelle utilise cette notation car il a constaté que dans la parole, l’intonation varie de façon continue (1897: 118) et les différentes notes musicales s’avèrent donc inadaptées pour représenter cette continuité :

\begin{abstract}
«La phrase musicale monte et descend par degrés préalablement déterminés d'une manière conventionnelle; la phrase parlée, partie d'une hauteur quelconque, parcourt d'un point à un autre une portion de l'échelle musicale, non par tons et par demi-tons, mais en suivant une progression qui paraît être le plus souvent régulièrement ascendante ou descendante ».
\end{abstract}

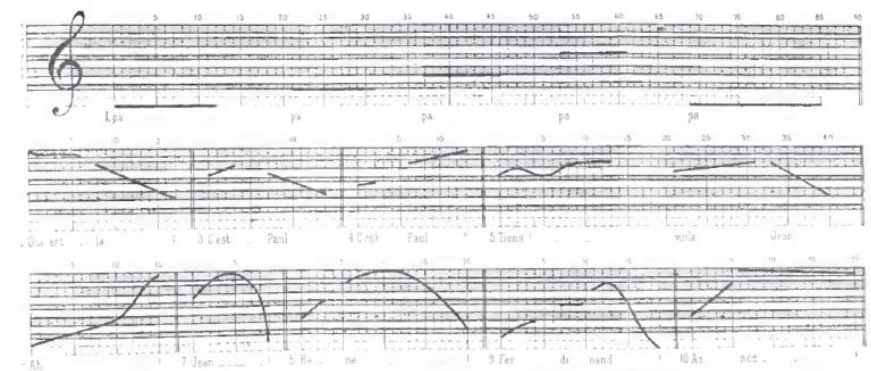

Fig. 3 : Courbe mélodique transcrite par Marichelle à partir du phonographe (1897, Planche 11).

Marichelle se montre très enthousiaste sur l'avenir de la transcription et des recherches sur l'intonation (Marichelle, 1897 : 121).

\begin{abstract}
«A propos de l'intonation, cette musique naturelle, nous venons de montrer comment il est possible de noter les inflexions profondes ou ténues de la voix: une ligne sinueuse se développant sur la portée et mesurant à la fois la hauteur musicale et le temps, c'est-à-dire l'intonation et le rythme. L'avenir est là, nul n'en saurait douter »
\end{abstract}

Il affirme qu'il serait nécessaire de mettre à contribution des maîtres de la parole (orateurs, acteurs et diseurs émérites), "qu'on les fasse parler devant la plaque vibrante comme on les fait poser devant l'objectif » et que la comparaison de ces enregistrements permettra de publier le premier traité d'intonation qui repose «sur des données certaines, sur des faits perceptibles » (Marichelle, 1897 : 120). Selon lui :

«La notation par lignes de la phrase parlée serait aussi lisible que celle de la phrase musicale par points. Cette lecture s'appuierait au début sur la répétition sonore du phonographe; de cette manière, l'image visuelle fournie par la courbe et l'image auditive ne tarderaient pas à se lier étroitement dans le rapport ordinaire du signe à la chose signifiée ».

\title{
5.2 Léonce Roudet ou l'application de la méthode graphique à l'étude de l'accentuation
}

Léonce Roudet avait, dès 1899, publié dans La Parole, un article - repris en 1910 dans ses Eléments de Phonétique Générale (1899 : 207) - préconisant une méthode expérimentale pour l'étude de l'accent. Son objectif est de proposer une nouvelle méthode permettant de déterminer l'intensité des sons du langage, en même temps que leur hauteur et leur durée fondée sur «les derniers progrès de la phonétique 
expérimentale » (1899 : 322). Dès le début de son article, il présente les trois paramètres constitutifs de l'accent, la hauteur, la durée et l'intensité, une décomposition que l'on retrouve encore dans les descriptions contemporaines de la prosodie. Roudet propose d'utiliser la méthode graphique pour représenter ces trois différents paramètres. Selon ce dernier, les mesures de durée sont simples à réaliser mais la délimitation des différents sons repose sur l'analyse de la forme des vibrations. La détermination de la hauteur est réalisée par le comptage des vibrations et la division de chacune de ces vibrations par leur durée, comme le faisait Marichelle. «Dans des recherches relatives à l'accent, où la hauteur a une importance considérable, j'ai cru devoir être plus exact et je mesure la hauteur musicale correspondant à chaque vibration » (1899 : 333). Il se pose d'ailleurs la question de savoir si cette précision n’est pas trop excessive, l'oreille ne percevant pas des variations de tonalité aussi faibles, mais c'est selon lui «le rôle de la science expérimentale de découvrir le détail des phénomènes que nos sens ne perçoivent qu'en bloc et dans leurs résultantes » (1899 : 334). A partir de ce tracé et d'un certain nombre de mesures, il va créer une représentation graphique représentant les résultats obtenus, en analysant, vibration par vibration la phrase « Petit, que fais-tu ? ». Concernant la représentation de la hauteur musicale, il s’inspire du système de transcription développé par Hector Marichelle, c'est-à-dire une portée composée de 10 lignes et de 9 niveaux de hauteur (cf. figure 4 ci-dessous). Elle est graduée selon l'axe temporel $(1,6 \mathrm{~mm}=1 \mathrm{cs})$ en abscisse ; en ordonnée est représentée la hauteur ${ }^{22}$.

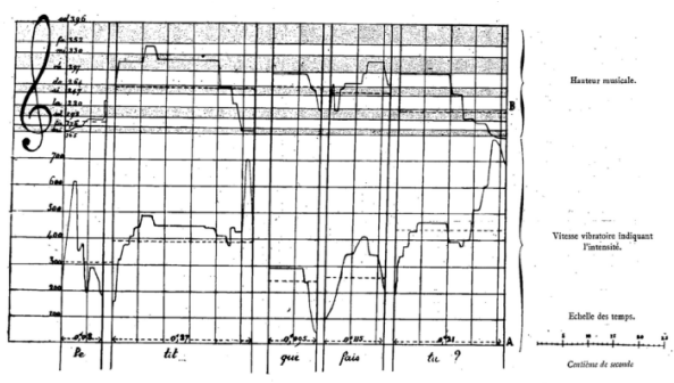

Fig. 4 : Système de transcription utilisé par Roudet pour l’étude de l’accent (1899 : 339).

La fréquence exacte des divers sons est reportée sur la portée musicale et les divers points reliés entre eux, ce qui fait apparaître une ligne mélodique. Roudet précise que sur la portée qu'il utilise, «il sera facile de représenter par une courbe continue les variations de hauteur » (1899: 339). Le mouvement mélodique de la parole devient alors mesurable. Pourquoi une telle précision est-elle devenue possible ? C’est grâce à l'introduction du tempérament égal ${ }^{23}$ ou gamme tempérée, avec une même distance entre chaque demi-ton, que les phonéticiens pouvaient compter sur un système mathématiquement calculable et invariable pour la représentation visuelle de l'intonation. On assiste à un phénomène comparable à l'introduction des barres de mesure régulières à l'âge baroque qui permettaient de calculer la longueur de chaque séquence et la place précise de l'accent.

En ce qui concerne l'accent, Roudet se concentre à la fin de son article sur les paramètres de hauteur et d'intensité, laissant provisoirement de côté « les rapports de durée qu'on a coutume d'étudier séparément sous le nom de quantité " (1899: 340) et "les variations continues de hauteur et d'intensité qui se produisent au cours d'une syllabe et que l'oreille ne perçoit guère que dans leur ensemble ». Il se focalise sur la hauteur et l'intensité moyenne de chaque syllabe (les traits horizontaux décrits plus haut). Après observation du tracé représenté sur la figure 4, Roudet affirme qu'en français, les variations d'intensité des syllabes sont liées à la fois aux mots employés et aux différentes combinaisons syntaxiques dans lesquelles ils peuvent entrer (1899 : 341). Les variations de hauteur, au contraire, sont liées aux différentes nuances de la pensée ou du sentiment, mais les deux types de variations peuvent interagir. Cependant :

«Il est utile de maintenir la distinction que je viens de faire et de se conformer ainsi

à la tradition des grammairiens qui, en général, réservent le nom d'accent à

l'accroissement d'intensité (ou de hauteur) de certaines syllabes liés aux mots et aux 
formes de phrases employées, et appellent toniques ou accentuées les syllabes ainsi mises en relief » (1899: 341).

Selon lui, chercher la place de l'accent, c’est déterminer les lois de répartition de l'intensité (ou de la hauteur) des syllabes d'après les formes verbales et syntaxiques de la langue étudiée. La méthode proposée par Roudet résout selon lui en partie la question de la nature de l'accent dans une langue donnée, en permettant de déterminer s'il s'agit d'un accent de hauteur ou d'intensité. Il mentionne également le problème de la mélodie du langage (en allemand "Sprachmelodik ») qui intéresse non seulement la phonétique et la linguistique, mais aussi la psychologie et même l'art oratoire et l'art dramatique. Comme Marichelle avant lui, Roudet se montre très enthousiaste à propos des progrès permis par la phonétique expérimentale et toutes les perspectives qu'elle apporte dans la description et la représentation de l'intonation. Et il conclut son article par cette très belle citation, qui résume en quelques lignes la rigueur et le labeur nécessaire à la pratique de cette toute nouvelle discipline que représente la phonétique expérimentale :

\begin{abstract}
«A devenir expérimentale, la Phonétique a gagné la certitude et la précision qui lui manquaient : la rançon de ces avantages, c'est un plus long et plus patient effort imposé au chercheur. Mais la plus humble parcelle de vérité scientifique a trop de prix pour qu'on regrette le labeur dépensé à la conquérir » (1899 : 344).
\end{abstract}

\title{
5.3 L'abbé Rousselot et le développement de la phonétique expérimentale
}

L’abbé Pierre Jean Rousselot, dans ses Principes de Phonétique Expérimentale (1908), se servira d’une technique analogue à celle de Roudet, moins précise bien que plus tardive. Rousselot fait la différence entre la hauteur physique d'un son qui résulte du nombre de ses vibrations et la hauteur psychologique, celle qui est perçue par l'oreille. Les deux ne correspondent pas forcément, comme l'avait déjà montré le système de tempérament égal (cf. 5.2.). Selon lui, il existe deux procédés pour déterminer la hauteur physique d'un son. Soit on mesure chaque période sous le microscope à l'aide d'un micromètre oculaire, soit on se contente de compter le nombre des vibrations sur le tracé pour un temps donné (à la façon de Marichelle et de Roudet). Il suffit ensuite de reporter toutes les variations de hauteur sur une portée musicale (cf. figure 5). Ce type de transcription de l'intonation s’inspire du système développé par Marichelle et utilisé par Roudet, à la différence près qu'elle est graduée en bandes équivalentes à un demi-ton, et non plus, en bandes de taille inégale correspondant soit à un ton, soit à un demi-ton. Cette équivalence le rend plus simple à lire, spécialement pour les non-musiciens. Quant à l’intensité, elle est indiquée en millimètres et correspond à l'amplitude mécanique des vibrations laryngées (ligne supérieure sur la figure 5) ; pour la durée, contrairement aux transcriptions de Marichelle et de Roudet, aucune unité de mesure n’est donnée, Rousselot reproduit simplement les enregistrements. Rousselot précise que «la durée se lit sur les tracés. Elle a pour mesure le son inscrit » (1908 : 990).

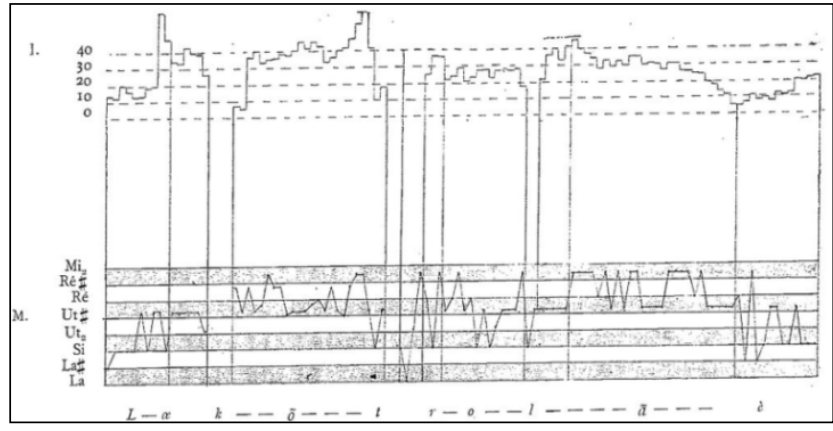

Fig. 5 : Système de transcription utilisé par Rousselot (1908 : 1005).

Malgré leurs efforts pour noter les paramètres prosodiques, ni Roudet, ni Rousselot, pourtant si précis, ne semblent accorder une grande importance à leur procédé de notation. Ils ne l'utilisent qu'à titre d'exemple, 
dans le but d'établir, sur certains points de la chaîne parlée, des relations entre intensité et hauteur musicale représentées sur un même schéma. Ainsi, la ligne mélodique qu’ils obtiennent en reliant les diverses notes apparaît étonnamment accidentée (en dents de scie). Chez Marichelle, la courbe est lissée et correspond davantage aux mouvements mélodiques tels qu'ils sont perçus par l'oreille. En cela, il se situe davantage dans la tradition des anciens grammairiens, alors que Roudet et Rousselot se positionnent dans une perspective plus expérimentaliste en prenant soin de décrire en détail l'évolution de l'intonation, dans ses moindres variations (ce que l'on appellerait aujourd'hui des variations micro-mélodiques). En réalité, il serait intéressant de visualiser deux courbes sur la même portée, l'une comportant l'ensemble de ces variations et l'autre, lissée, correspondant à l'évolution de la hauteur telle que l'oreille la perçoit.

\section{Discussion}

Au cours des siècles, nous avons montré que différentes approches se sont succédées, privilégiant tour à tour, soit une vision globale, soit une vision détaillée des phénomènes prosodiques. Ainsi Meigret s'intéresse à la prosodie affectant des unités de plus grande taille telles que les phrases ou les propositions, alors que les grammairiens du XVIIe siècle se concentrent presque exclusivement sur la quantité, c’est-à-dire la durée qui est décrite par le rythme musical (les noires et les blanches correspondant à des syllabes courtes ou longues). Cette approche extrêmement focalisée va s'élargir au siècle suivant lorsque les auteurs vont distinguer de façon plus fine le rythme de l'accentuation. Au cours du même siècle, la démarche des encyclopédistes initie une volonté d’exhaustivité qui consiste à répertorier toutes les connaissances sur les différents domaines dans leur complexité. Cette approche va aboutir au siècle suivant à la mise au point des méthodes expérimentales scientifiques qui permettront, grâce à l’instrumentation, une précision extrême.

Par ailleurs, la description des différents auteurs va varier en fonction de leur méthodologie. Dans un premier temps, les grammairiens, qui ne disposaient pas d'appareillage permettant de mesurer les phénomènes relatifs à la production de la parole, étaient obligés de fonder leurs analyses sur l'autoperception. L'oreille était donc un outil de première importance dans l'analyse de la prosodie. En effet, c'est la sensation qui va primer et déterminer le choix du mode de notation. La prosodie étant perçue comme continue par l'oreille, il est tout-à-fait naturel que les auteurs aient adapté la notation musicale pour la transcrire.

L’apparition des premiers appareillages et de la méthode graphique au XIXe siècle va bouleverser la recherche au niveau de la description de la parole. Dorénavant, ils vont être capables de visualiser les phénomènes vibratoires propres à la production de la parole sous une forme graphique, mais pour obtenir le tracé propre à la mélodie de la parole, ils vont devoir calculer, période après période, la correspondance en fréquence qu'ils vont reporter point par point sur une portée musicale. Le but de l'étude ne change pas : on veut toujours repérer et décrire la production des phénomènes phonétiques. Pourtant, la notation musicale obtenue à partir des appareillages ne correspond plus à une description basée uniquement sur la perception, mais à la production de la parole décrite de façon très détaillée grâce à l'instrumentation (ce qui annonce déjà les nouvelles méthodes expérimentales). Chez Rousselot et chez Roudet, cette représentation extrêmement fine reflète leur volonté de décrire le plus exhaustivement possible le signal de parole grâce à un ensemble de mesures acoustiques et articulatoires. En revanche, Marichelle adopte une position intermédiaire car, tout en partant de la même technique, il va reproduire des contours lissés pour obtenir un résultat correspondant à ce qu'il perçoit, c'est-à-dire une modification continue et progressive de la hauteur en fonction du temps.

\section{Conclusion}

La transcription musicale, utilisée pendant plusieurs siècles, nous semble très intéressante, car elle permet de transcrire graphiquement de manière facilement accessible les phénomènes prosodiques. Il n’est alors pas étonnant qu'elle anime l'imagination des grammairiens et qu'elle s'adapte facilement à différents courants de pensée. Cependant, aucun des modèles utilisés n’est capable de reproduire de manière 
satisfaisante les différents paramètres prosodiques de la parole. Ainsi, les intervalles de la gamme ne sont pas assez fins pour reproduire les variations de hauteur propres au ton laryngien, et inversement, les courbes établies à l'aide de l'instrumentation sont trop détaillées pour qu'on puisse reproduire facilement la mélodie d'une phrase donnée. C’est ainsi qu’à partir d'une époque donnée, la transcription musicale ne va plus s'avérer suffisante. Il faut alors admettre qu'au bout du compte, un facteur essentiel distingue la mélodie de la langue de la mélodie musicale : alors que la variation de hauteur est continue dans la parole, elle est discontinue dans la musique occidentale. Quant au rythme de la langue parlée, il ne peut pas être transcrit dans le cadre du système de notation classique en vigueur en musique. De façon générale, la transcription musicale de la prosodie est un bon exemple qui permet de comprendre l'évolution des idées et de la description de la langue française. Les progrès techniques qui ont permis la naissance de la phonétique expérimentale en tant que science à part-entière, ont abouti à un niveau de description très fin de la parole. Cependant, nous pouvons déplorer qu'en chemin, on ait perdu le lien direct entre la perception de l'auditeur et sa visualisation assurée par la transcription musicale.

\section{Références bibliographiques}

Bacilly, B. de (1668). Remarques curieuses sur l'art de chanter, et particulièrement pour ce qui regarde le chant français, Paris. Edition utilisée : Traité de la methode ou art de bien chanter, par le moyen duquel on peut en peu de temps se perfectionner dans cet Art, \& qui comprend toutes les Remarques curieuses que l'on y peut faire, Paris, G. de Luyne, $1671^{2}$.

Beauzée, N. (1767). Grammaire générale ou exposition des éléments nécessaires du langage pour servir de fondement à l'étude de toutes les langues, vol. 1, Paris.

Bosquet, J. (1586). Elemens ou institutions de la langue française, Mons ca. 1586. Grand Corpus des grammaires françaises, des remarques et des traités sur la langue (XIVe-XVIIe s.), Bernard Colombat, Jean-Marie Fournier (dir.), Paris, Classiques Garnier Numérique, 2011.

Buffet, M. (1668). Nouvelles Observations sur la langue françoise, Paris.

D'Olivet, P.J. (1736). Traité de la prosodie française, Paris.

Diderot, D. et d'Alembert, J. (ed. 1751-1772). Encyclopédie ou dictionnaire raisonné des sciences, des arts et des métiers. Paris, Briasson, David e. a.

GGR (1660). Grammaire générale et raisonnée contenant les fondemens de l'art de parler, expliqués d'une manière claire et naturelle par Antoine Arnauld et Claude Lancelot, Paris. Reprint de la $3^{\mathrm{e}}$ éd., 1676, Stuttgart-Bad Cannstatt, Fr. Fromman Verlag, 1966.

Grimarest, J. L. Le Gallois de (1707). Traité du récitatif dans la lecture, dans l'action publique, dans la déclamation, et dans le chant ; avec un traité des accens, de la quantit, \& de la prononciation.

Janet, P. (1887). Les Origines de la Philosophie d'Auguste Comte - Comte et Saint-Simon. Revue des Deux Mondes, 82.

Lamy, B. (1676). De l'Art de parler, Paris. Reprint Munich, Wilh. Fink, 1980.

Loulié, E. (1696). Éléments ou principes de musique, mis dans un nouvel ordre. Paris.

Marichelle, H. (1897). La Parole d'après le Tracé du Phonographe. Paris : Delagrave.

Meigret, L. (1550). Le Tretté de la Grammaire française. Paris.

Ramus, P. (1572). Grammaire, Paris. Grand Corpus des grammaires françaises, des remarques et des traités sur la langue (XIVe-XVIIe s.), Bernard Colombat, Jean-Marie Fournier (dir.), Paris, Classiques Garnier Numérique, 2011, consulté le 14 juin 2015.

Raparlier (1772). Principes de musique, les agréments du chant et un essai sur la prononciation, l'articulation et la prosodie de la langue françoise, Lille.

Roudet, L. (1899). Méthode expérimentale pour l’étude de l’accent. La Parole, 5, 321-344.

Rousselot, l’abbé (1908). Principes de Phonétique Expérimentale. Paris : Welter, 1901-1908, 2e vol. 
Tyard, P. de (1555). Solitaire second ou Prose de la musique, Lyon.

Saint-Lambert (1702). Les Principes du clavecin, Paris. Reprint Genève Minkoff, 1972.

Vairasse d'Allais, D. (1681), Grammaire Méthodique contenant en abrégé les Principes de cet art et les règles les plus nécessaires à la langue française, Paris. Grand Corpus des grammaires françaises, des remarques et des traités sur la langue (XIVe-XVIIe s.), Bernard Colombat, Jean-Marie Fournier (dir.), Paris, Classiques Garnier Numérique, 2011, consulté le 14 juin 2015.

Vaissière, J. (1997). Langage, prosodie et syntaxe. Traitement Automatique des Langues, ATALA, 38.1 : 53-82.

\footnotetext{
${ }^{1}$ Nous ne traitons pas ici le vers mesuré à l’Antiquité et sa transcription musicale, mais la description du français comme il figure chez les grammairiens et les théoriciens du chant français.

${ }^{2}$ En vérité, déjà l'utilisation de notes précises d'une seule octave remet en cause l'objectif apparent de l'auteur : il existe bien des voix plus graves, plus aiguës... Il apparaît qu'il faut aller au-delà d'une lecture véritablement musicale.

${ }^{3}$ Du XIIe au XVIe siècle, l'habitude d'utilisation de notes altérées, non prévues dans l'échelle diatonique des hexacordes, mais repérables dans leur contexte musical selon un certain règlement codifié.
}

${ }^{4}$ Du latin punctum contra punctum, c.à.d. point contre point, phrase contre phrase ou note contre note. Principe de composition généralement en usage pour les œuvres entières jusqu'à la fin du XVIe siècle, puis dans des formes particulières comme le ricercar ou la fugue jusqu'à aujourd'hui.

${ }^{5}$ Notamment vers la fin du siècle, on note l'émergence d'une conception de la simultanéité des sons qui se développera vers la pensée harmonique à la fin du siècle entrainnant finalement une accentuation plus régulière et plus fréquente de la musique (voir infra).

${ }^{6}$ Le chromatisme désigne un système musical qui se fonde sur la division de l'octave en douze parties égales. Depuis le XIVe siècle, ce terme est utilisé pour désigner les altérations chromatiques d'un demi-ton qui, dans la musique de la fin du XVIe siècle, prennent de plus en plus d'importance pour la tension expressive intrinsèque à la musique, car chaque note, étrangère à l'harmonie avec laquelle elle retentit, demande inévitablement d'être résolue.

${ }^{7}$ Effectivement, pour Bosquet (1586: 151), l'accent circonflexe s'utilise soit pour indiquer, selon la tradition grecque, un mouvement montant, puis descendant de la voix, soit pour indiquer un son long.

${ }^{8}$ Le concept du phonème n’étant pas encore développé, la référence de toute réflexion est la lettre.

${ }^{9}$ Terme de la notation mesurée des XVe et XVIe siècles qui correspond à la codification de l'accélération ou du ralentissement de l'exécution à la base de rapports numériques.

${ }^{10}$ On pense au récitatif de Quinault/Lully qui se développe pendant ces années-là.

${ }^{11}$ La quantité désigne la différence entre des syllabes longues ou brèves à cause de la durée du noyau vocalique.

${ }^{12}$ Voir la régularité du nombre des mesures qui composent une phrase musicale ou les parties des danses.

${ }^{13}$ Il s'agit d'un problème typique pour le chant: si le $e$ muet à la fin des mots féminins détermine facilement la quantité de la dernière syllabe, celle-ci est beaucoup moins évidente pour les mots avec une terminaison masculine. L'auteur traite alors les différentes lettres qui peuvent terminer un mot masculin.

${ }^{14}$ Voir par exemple GGR (1660 : 16-17) ou Vairasse d'Allais (1681 : 38-39).

${ }^{15}$ On passe d'une musique d'un concept linéaire à une pensée en accords, basée sur la progression de la basse, définie comme porteuse de l'harmonie.

${ }^{16}$ Vairasse d'Allais (1681 : 36-37) distingue des syllabes très-brèves (avec $e$ muet), brèves (les syllabes indifférentes le sont «par nature »), longues (possible «par position ») et très-longues («par position \& par accent »).

${ }^{17}$ Chez Saint-Lambert (1702) par exemple on lit une longue réflexion sur la difficulté notamment des gens en province, dans l'impossibilité d'écouter les bons musiciens professionnels, de trouver le juste tempo d'une pièce.

${ }^{18}$ Il présente son système de transcription dans le chapitre 5 « De l'intonation » de son ouvrage « La Parole d'après le tracé du Phonographe » (1897) préfacé par Marey lui-même. Selon lui, «l'étude de l'intonation sans le secours des instruments, présenterait des difficultés à peu près insurmontables » $(1897: 111)$. 


\footnotetext{
${ }^{19}$ En musique, désigne un intervalle désigne un écart de hauteur entre deux notes. En acoustique, il correspond à un rapport de fréquence entre deux sons.

${ }^{20}$ Étymologie. 1903, Colette, in T. L. F. ; de glisser, sur le modèle des mots ital. en -ando.

${ }^{21}$ Le terme, déjà présenté comme entrée dans le dictionnaire de musique allemand Handlexikon der Tonkunst d'Oscar Paul (Leipzig 1870-1873) ne figure en France qu'à partir de 1903 (voir le TLF). Le terme glissando est expliqué par Paul comme suivant : "gleiten, eine Untertasten-Passage auf dem Clavier, wenn sie mit einem Finger ausgeführt werden soll », le livre complet : https://archive.org/details/handlexikondert00paulgoog

${ }^{22}$ Pour ce paramètre, la gradation correspond à la fréquence de chaque note de la gamme tempérée $(\mathrm{Mi} 2=165 \mathrm{~Hz}$, $\mathrm{Fa} 2=175 \mathrm{~Hz}$, etc.). Des traits horizontaux sont tracés à l'endroit où se situe chaque note ; la distance entre chaque ligne de la portée ainsi obtenue est donc inégale, puisqu'elle correspond à des intervalles de fréquence inégaux, mais elle est "proportionnelle au logarithme des fréquences vibratoires, car c'est cela que notre oreille apprécie " (Roudet, 1899 : 207).

${ }^{23}$ Le tempérament égal a été mentionné pour la première fois chez Werkmeister en 1688. Théoriquement connu depuis longtemps, il ne sera généralisé qu’au XIXe siècle.
} 